\title{
Erratum to: Determinants of ante-partum depression: a multicenter study
}

\author{
Matteo Balestrieri · Miriam Isola • Giulia Bisoffi - Salvatore Calò • \\ Anita Conforti - Lorenza Driul • Diego Marchesoni - Paola Petrosemolo · \\ Michela Rossi - Adriana Zito - Stefania Zorzenone - Guido Di Sciascio • \\ Roberto Leone $\cdot$ Cesario Bellantuono
}

Published online: 3 August 2012

(c) Springer-Verlag 2012

\section{Erratum to: Soc Psychiatry Psychiatr Epidemiol \\ DOI 10.1007/s00127-012-0511-z}

Due to an unfortunate error, the names of all authors have been published in the incorrect order both in the author list and affiliation of the original publication. The corrected author list is given below:

Matteo Balestrieri, Miriam Isola, Giulia Bisoffi, Salvatore Calò, Anita Conforti, Lorenza Driul, Diego Marchesoni, Paola Petrosemolo, Michela Rossi, Adriana Zito, Stefania Zorzenone, Guido Di Sciascio, Roberto Leone, Cesario Bellantuono.

The online version of the original article can be found under doi:10.1007/s00127-012-0511-z.

M. Balestrieri · S. Zorzenone

Section of Psychiatry, Department of Experimental and Clinical Medical Sciences, University of Udine, Udine, Italy

M. Balestrieri $(\square)$

Clinica Psichiatrica, Azienda Ospedaliero-Universitaria,

P.le S. M. Misericordia 15, 33100 Udine, Italy

e-mail: matteo.balestrieri@uniud.it

M. Isola

Department of Medical and Morphological Sciences,

University of Udine, Udine, Italy

G. Bisoffi

Biostatistics Office, University Hospital, Verona, Italy

S. Calò · A. Zito · G. Di Sciascio

Department of Neurological and Psychiatric Sciences,

University Hospital "Policlinico Consorziale",

University "Aldo Moro", Bari, Italy
A. Conforti $\cdot$ R. Leone

Clinical Pharmacology Unit, University of Verona, Verona, Italy

L. Driul · D. Marchesoni

Clinic of Obstetrics and Gynaecology, University Hospital

of Udine, Udine, Italy

P. Petrosemolo

Section of Psychiatry and Clinical Psychology,

Department of Medicine and Public Health,

University of Verona, Verona, Italy

M. Rossi · C. Bellantuono

Psychiatric Unit, Department of Neuroscience,

Polytechnic University of Marche, Ancona, Italy 